\title{
THE DRAG REDUCTION OF MICROCHANNEL FLOW BY CONTRAST WETTABILITY
}

\author{
Elizaveta Ya. Gatapova ${ }^{1, *}$, and Dmitry S. Gluzdov ${ }^{1,2}$ \\ ${ }^{1}$ Kutateladze Institute of Thermophysics SB RAS, 630090 Novosibirsk, Russia \\ ${ }^{2}$ Novosibirsk State University, 630090 Novosibirsk, Russia
}

\begin{abstract}
The model is developed and the boundary condition is approximated at the lower boundary of the channel. It is shown that a hydrophobic surface can significantly reduce the hydraulic resistance due to the occurrence of bubble layer. Moreover, at the slip ratio equal to 1 $\mathrm{mm}$, the water flow rate in the channel with the height of $1 \mathrm{~mm}$ increases by 2.5 times.
\end{abstract}

\section{Introduction}

The endeavor of manufacturers to increase the processors speed requires the reduction of their sizes that inevitably leads to problems associated with heat transfer. Contemporary liquid cooling systems are effectively used in combination with the microchannel structure. At that, the smaller the microchannel thickness, the more efficient is heat removal. However, the reduction of the microchannel thickness leads to a strong increase in the hydraulic resistance that substantially prevents heat transfer and results in the necessity to maintain high pressure drops at the microchannel inlets and outlets [1,2]. This, in turn, affects the size of the entire cooling system.

It is clear that the further improvement of advanced liquid cooling systems is associated with the problem of reducing the hydraulic resistance in microchannels. One of the previously proposed solutions to this problem is the application of additional microstructure on the surface of the microchannels [3]. The essence of this solution lies in the fact that the additional microstructure of the microchannel contains gas, which does not hinder the liquid when contacting with the applied surface, unlike the microchannel walls, to which liquid is adhered as consistent no-slip boundary condition [4].

This technique may allow reducing the hydraulic resistance of up to $40 \%$ [5]. However, it has drawbacks related to the high cost of improved microchannel manufacturing, as well as to the problem of keeping gas within the microstructure [6], maintaining flow stability [7], and preventing rupture of a liquid film [8,9].

Another solution to the problem of high hydraulic resistance in a microchannel is proposed in [10]. It was revealed that on the microchannel surface coated with a hydrophobic material, a bubble layer is formed. We proposed to use this effect to ensure liquid slippage at the surface of local microchannel sections that will allow reducing

Corresponding author: egatapova@gmail.com 
hydraulic resistance. The application of contrast hydrophobic coating can enhance heat transfer and improve the liquid pumping conditions in general.

The results obtained previously in [10], support further research prospects in this area. The aim of this work is to study the flow characteristics in a microchannel with applied contrast hydrophobic coating through a numerical solution of the two-dimensional system of Navier-Stokes equations with a singularity in the boundary condition at the lower boundary.

\section{Formulation of a problem}

A tentative diagram of the problem is shown in fig. 1. The lower wall of the microchannel with specified length and height is coated with contrast hydrophobic surface to form bubble layer. The distribution of velocity and pressure at the inlet to the microchannel, as well as all the parameters of the flowing liquid, such as viscosity and density, are known. The liquid is considered incompressible. Water served a working liquid.

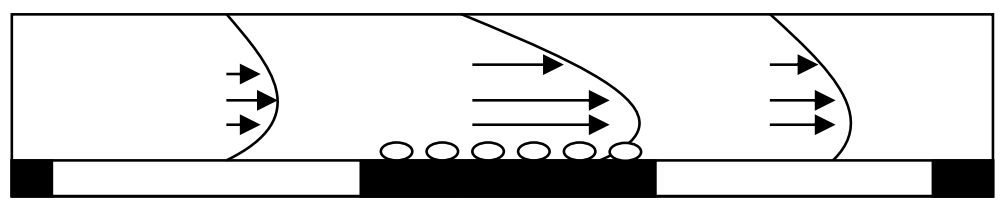

Fig. 1. Visualization of the problem.

It is required to find velocity and pressure fields in the microchannel through numerical solution of the Navier-Stokes equations.

To obtain a complete system of the Navier-Stokes equations (1), we use at the lower boundary the conventional Navier condition with the feature in the $\beta$ function, which takes its maximum value on the surfaces with a hydrophobic coating and becomes zero on a conventional microchannel surface. On the boundary between the regular and hydrophobic surfaces, the $\beta$ function is smoothed.

$$
\left\{\begin{array}{l}
\frac{\partial u}{\partial t}+u \frac{\partial u}{\partial x}+v \frac{\partial u}{\partial y}=-\frac{1}{\rho} \frac{\partial P}{\partial x}+\eta\left(\frac{\partial^{2} u}{\partial x^{2}}+\frac{\partial^{2} u}{\partial y^{2}}\right)+g \sin \alpha \\
\frac{\partial v}{\partial t}+u \frac{\partial v}{\partial x}+v \frac{\partial v}{\partial y}=-\frac{1}{\rho} \frac{\partial P}{\partial y}+\eta\left(\frac{\partial^{2} v}{\partial x^{2}}+\frac{\partial^{2} v}{\partial y^{2}}\right)+g \cos \alpha \\
\frac{\partial u}{\partial x}+\frac{\partial u}{\partial y}=0 \\
u=\beta(x) \frac{\partial u}{\partial y}
\end{array}\right.
$$

Here $u$ and $v$ - are the horizontal and vertical velocity components, respectively; $\eta$ - is the kinematic viscosity.

The boundary condition for the horizontal velocity component on the upper wall corresponds to the conventional no-slip boundary condition, while the condition on the lower wall is approximated by formula (2) using the technique shown below. For the vertical velocity we use the impermeability condition. In other cases, where the boundary condition is needed in the numerical scheme, we use the so-called "soft conditions", i.e. the 
first derivative of a sought quantity is made equal to zero. Function $\beta(x)$ (2) has the following configuration (fig. 3).

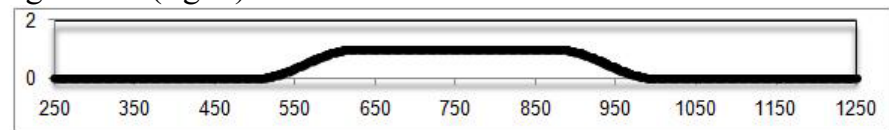

Fig. 2. Approximation of $\beta(x)$ function.

$$
\beta=\left\{\begin{array}{c}
\beta_{\max }\left[\frac{1+\sin \left(\pi \frac{x-l_{i+1}}{\Delta l}-\frac{\pi}{2}\right)}{2}\right], x \in\left[l_{i+1} ; l_{i+1}\right] \\
\beta_{\max }\left[\frac{1-\sin \left(\pi \frac{x-l_{i+2}+\Delta l}{\Delta l}-\frac{\pi}{2}\right)}{2}\right], x \in\left[l_{i+2}-\Delta l ; l_{i+2}\right]
\end{array}\right.
$$

where $\beta_{\max }$ - is the maximum value of the slippage coefficient, $l$ - is the length of the section with hydrophobic coating, $\Delta l$ - half of the section, on which the function is smoothed, $i-$ is the number of a section with hydrophobic coating.

\section{Results}

The problem is solved for the Poisson flow of the water at the section with the bubble layer, pressure gradient $\partial \mathrm{P} / \partial \mathrm{x}=0.1 \mathrm{~atm} / \mathrm{m}$, dynamic viscosity $\mu=0.001 \mathrm{~kg} / \mathrm{m}^{*} \mathrm{~s}$, the characteristic bubble size $\delta=1 \mu \mathrm{m}$, and the slippage length $\beta=1 \mathrm{~mm}$. We have revealed that the flow rate has increased 2.5 times in comparison with that for the section without bubble layer (fig.3).

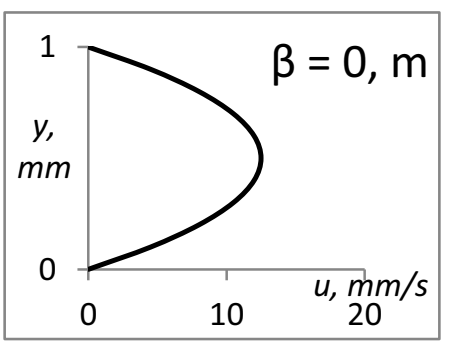

a

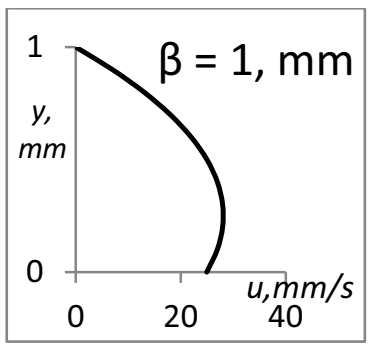

b

Fig. 3. The velocity profile: a - section with the conventional surface; $b$ - section with the hydrophobic surface. 


\section{Conclusions}

A model was developed and the boundary condition was approximated at the lower boundary of the channel. It is shown that a hydrophobic surface can significantly reduce the hydraulic resistance due to the occurrence of bubble layer. Moreover, at the slip ratio equal to $1 \mathrm{~mm}$, the water flow rate in the channel with the height of $1 \mathrm{~mm}$ increases by 2.5 times.

\section{Acknowledgments}

The work was supported by the Russian Science Foundation (project No. 14-19-01755).

\section{References}

1. T.M. Squires and S.R. Quake, Rev. Mod. Phys. 77, 977 (2005)

2. H.A. Stone, A.D. Stroock, A. Ajdari, Annual Reviews of Fluid Mechanics 36 (2004)

3. J. Ou, B. Perot, and J. P. Rothstein, Phys. Fluids 16 (2004)

4. E. Karatay, A.S. Haase, C.W. Visser, C. Sun, D. Lohse, P.A. Tsai, R.G.H. Lammertnik, Proc. Nat. Acad. Sci. 110, 8422 (2013).

5. J.P. Rothstein, Annual Reviews of Fluid Mechanics 42, 89 (2010)

6. D. Byun, J. Kim, H.S. Ko, H.C. Park, Phys. Fluids 20, 113601 (2008)

7. V.S. Ajaev, E.Ya. Gatapova, O.A. Kabov, Phys. Fluids 25, 122102 (2013)

8. D.V. Zaitsev, O.A. Kabov, V.V. Cheverda, N.S. Bufetov, High Temperature 42, 3 (2004)

9. D.V. Zaitsev, D.P. Kirichenko, O.A. Kabov, Tech. Phys. Lett. 41, 6 (2015)

10. E.Ya. Gatapova, V.S. Ajaev, O.A. Kabov, JETP Lett. 101, 3 (2015) 